\title{
ANÁLISE DO ESCOAMENTO E TRANSFERÊNCIA DE CALOR EM UM LEITO FIXO A PARTIR DA TÉCNICA DE FLUIDODINÂMICA COMPUTACIONAL
}

\author{
U. J. CASTRO*, R. BÉTTEGA, J. T. FREIRE \\ Universidade Federal de São Carlos, Departamento de Engenharia Química \\ "e-mail: ubiranilsoncastro@gmail.com.br
}

\begin{abstract}
RESUMO
A operação de secagem é amplamente utilizada na indústria química, devido a necessidade de manutenção de características dos materiais e conservação de suas propriedades em diversos processos. Pesquisadores empregam a abordagem em camada fina para simplificar algumas condições e facilitar a obtenção de dados para projeto de secadores, no entanto, as considerações utilizadas são frequentemente questionadas quanto a sua validade. A Fluidodinâmica Computacional (CFD - Computational Fluid Dynamics) é uma técnica poderosa para a solução numérica de problemas de transferência de quantidade de movimento, calor e massa. Neste trabalho são apresentados resultados simulados a partir de um modelo bidimensional utilizando-se CFD, incorporando diferentes perfis para a distribuição radial da porosidade, em regime transiente. Os resultados da distribuição da velocidade e temperatura de um leito fixo aquecido pelo ar são apresentados considerando a dinâmica de aquecimento do meio. Ao comparar-se os resultados obtidos através do modelo com dados experimentais presentes na literatura, verificou-se a capacidade do modelo e procedimento numérico adotados para representação do fenômenos envolvidos. Os resultados corroboram na comprovação da influência da distribuição porosidade na dinâmica de aquecimento, resultando em significativas variações de velocidade e temperatura no processo.
\end{abstract}

\section{INTRODUÇÃO}

Das diversas operações industriais no campo da Engenharia Química a secagem sobressai-se por ser um dos métodos mais antigos de conservação de alimentos, consistindo na redução do teor de umidade para um nível que permite o armazenamento seguro durante um período prolongado. Além disso, a redução do volume do produto facilita seu manuseio, transporte e armazenamento.

$\mathrm{Na}$ secagem em leito fixo geralmente o ar de secagem aquecido é posto em contato com material particulado úmido acondicionado em uma coluna vertical de geometria cilíndrica, a injeção do ar é realizada na base do sistema e percola o leito até o topo, saindo saturado pela umidade retirada no processo.

A ocorrência de restrição estrutural presente em leitos fixos ensejou a investigação de formas indiretas de medida experimental das variáveis neste equipamento, tais como velocidade, temperatura e umidade.

Durante décadas a técnica de avaliação da cinética de secagem conhecida como camada fina vem sendo utilizada por pesquisadores para avaliar a cinética dos mais diversos materiais. Para que um determinado sistema possa ser englobado em tal abordagem, o tempo de residência do gás no 
sistema deverá ser curto o bastante para que se possa considerar que o equilíbrio térmico e mássico entre as fases seja atingido rapidamente e a variação de umidade e temperatura da fase gasosa na camada delgada seja desprezível. Assim, é possível assumir que uma camada de material a ser seco é fina o bastante a fim de assegurar que gradientes de temperatura e umidade no interior do material possam ser considerados desprezíveis.

Trabalhos recentes da literatura amiúde vêm questionando a metodologia adotada para a camada fina e consequentemente os resultados obtidos através dela. Apesar emprego de medidas indiretas há diversos trabalhos da literatura que conseguem descrever a curva de secagem para as condições do experimento a partir da abordagem em camada fina, entretanto, esta não fornece indicação sobre o transporte de energia e de umidade no interior do meio poroso (PERAZZINI, 2014).

Estudos realizados na estrutura deste sistema já comprovaram que a porosidade apresenta um comportamento oscilatório na direção radial. A porosidade afeta diretamente a velocidade de escoamento do fluido através do leito, que por sua vez está intimamente ligada à transferência de calor e massa.

Neste contexto, a utilização cada vez mais intensa de metodologias e ferramentas de simulação é um dos pontos-chave para o sucesso de engenheiros envolvidos em processo industriais. De forma complementar a outros métodos de análise, tais como técnicas experimentais laboratoriais ou ensaios em planta piloto, as técnicas de dinâmica dos fluidos computacional ou CFD (Computational Fluid Dynamics), ganharam importância estratégica para a pesquisa e desenvolvimento de processos.

A partir desta técnica, é possível obter informações detalhadas sobre 0 comportamento da transferência de quantidade de movimento, distribuição de calor e massa em um processo de secagem.

Nagliate et al. (2010) realizaram a simulação da fluidodinâmica em um leito fixo empregando CFD obtendo respostas coerentes com os dados experimentais. Um avanço posterior foi a simulação do escoamento e da transferência de calor obtida por Béttega et al. (2013) que inclui perfis de porosidade radial, estudando um leito fixo em regime permanente de temperatura, devido o aquecimento pela parede. Entretanto, não existem estudos que simulem a transferência de calor e massa em leito fixo em regime transiente, apesar da presença de trabalhos que simulam com sucesso a transferência de calor e massa para outros sistemas (KRAWCZYK; BADYDA, 2011).

Desta forma, o trabalho propõem-se avaliar o processo de secagem em leito fixo utilizando a técnica de CFD (software ANSYS - FLUENT), com o propósito de estudar os fenômenos de transferência de calor, investigando o modelo de abordagem em camada fina. Este estudo possibilitará responder questões acerca da dinâmica do processo de secagem em leito fixo, além de gerar informações detalhadas acerca da distribuição de velocidade e temperatura no interior do meio poroso. Realizando ainda a verificação dos resultados simulados com dados apresentados por Perazzini (2014) de medidas experimentais.

\section{MATERIAIS E MÉTODOS}

Os dados experimentais utilizados para análise, assim como as condições geométricas e operacionais do equipamento, propriedades do sólido e do ar de secagem empregadas na simulação dos resultados foram baseados no trabalho de Perazzini (2014). 


\subsection{Modelagem Matemática e Solução Numérica}

Para as simulações realizadas neste trabalho adotou-se um modelo bidimensional, considerando a axissimetria do sistema, que engloba fenômenos acoplados de transferência de calor e quantidade de movimento. As simulações foram realizadas considerando o regime transiente, pois ar aquecido perpassa o meio poroso possibilitando a transferência de calor e elevação na temperatura deste com o tempo, as propriedades do fluido e do sólido permanecem constantes. Maiores informações sobre o equacionamento podem ser encontradas em ANSYS (2009).

Para a simulação numérica do transporte de quantidade de movimento as equações de Navier-Stokes para o escoamento monofásico de fluidos são adotadas, incluindo-se um termo fonte que represente as perdas viscosas e inerciais devido à presença do meio poroso. Para o caso do escoamento de ar estudado neste trabalho, o termo fonte toma a forma da Equação 1:

$$
S_{i}=-\left(\frac{\mu}{\alpha(r)} v_{i}+C_{2}(r) \frac{1}{2}|v| v_{i}\right)
$$

Na equação 1, a permeabilidade do leito $(\alpha)$, bem como o coeficiente que engloba as perdas inerciais no sistema $\left(\mathrm{C}_{2}\right)$, foram obtidos a partir das Equações 2 e 3, respectivamente:

$$
\begin{aligned}
& \alpha(r)=\frac{D_{p}^{2}}{150} \frac{\varepsilon^{3}}{(1-\varepsilon)^{2}} \\
& C_{2}=\frac{3,5}{D_{p}} \frac{(1-\varepsilon)}{\varepsilon^{3}}
\end{aligned}
$$

Em alguns casos a porosidade do sistema foi estimada a partir dos modelos de
Haideger et al. (1989), Giudici (1991) e Mueller (1992).

As condições de contorno adotadas para a solução do modelo foram:

- Na face de entrada de ar no leito, adotou-se a condição de velocidade do ar prescrita, 0,98 $\mathrm{m} \mathrm{s}^{-1}$, com ar entrando a uma temperatura de $338,15 \mathrm{~K}$;

- Na parede a condição de não deslizamento foi adotada;

- Eixo de axissimetria foi incorporado ao sistema, possibilitando a simulação do problema em duas dimensões;

- Na saída, utilizou-se a condição de pressão prescrita.

O domínio computacional empregado nas simulações no presente trabalho foi constituído das três regiões conforme ilustrado na Figura 1. Estas regiões foram definidas no domínio computacional com objetivo de captar efeitos tanto internos do meio poroso, quanto na entrada e saída do equipamento. A região de entrada foi definida $4 \mathrm{~cm}$ de comprimento, o leito fixo propriamente dito com $5 \mathrm{~cm}$, região de saída com $5 \mathrm{~cm}$ para um diâmetro constante de 10 $\mathrm{cm}$. Demais parâmetros adotados na solução deste modelo são apresentados na Tabela 1 .

Figura 1 - Representação esquemática de um leito fixo.

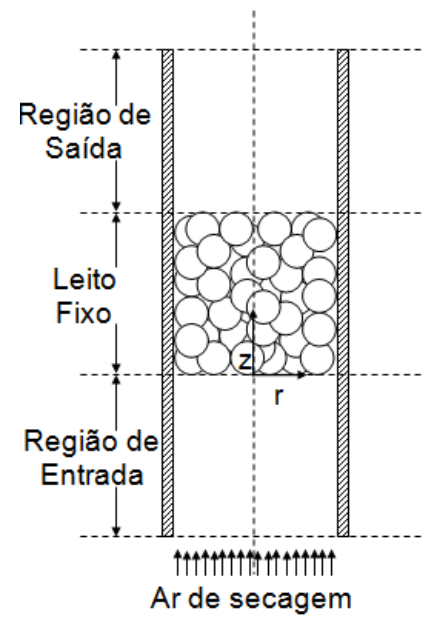

Fonte: Autor (2015) 
Tabela 1 - Características e parâmetros do modelo.

\begin{tabular}{cc}
\hline Parâmetro & Valor \\
\hline $\mathrm{k}_{\mathrm{eff}}$ & $0,27 \mathrm{~W} \mathrm{~m}^{-1} \mathrm{k}^{-1}$ \\
$\mathrm{D}$ & $0,10 \mathrm{~m}$ \\
$\mathrm{D}_{\mathrm{p}}$ & $2,810^{-3} \mathrm{~m}$ \\
$\rho_{\mathrm{p}}$ & $2502 \mathrm{~kg} \mathrm{~m}^{-3}$ \\
$\mathrm{Cp}_{\mathrm{p}}$ & $1720 \mathrm{~J} \mathrm{~kg} \mathrm{~K}^{-1}$ \\
$\rho_{\mathrm{ar}}$ & $1,225 \mathrm{~kg} \mathrm{~m}^{-3}$ \\
$\mathrm{C}_{\mathrm{ar}}$ & $1006,43 \mathrm{~J} \mathrm{~kg}^{-1} \mathrm{~K}^{-1}$ \\
$\mathrm{k}_{\mathrm{ar}}$ & $0,0242 \mathrm{~W} \mathrm{~m}^{-1} \mathrm{~K}^{-1}$ \\
$\mu_{\mathrm{ar}}$ & $1,789410^{-5} \mathrm{~kg} \mathrm{~m}^{-1} \mathrm{~s}^{-1}$ \\
\hline
\end{tabular}

Fonte: Autor (2015)

A obtenção da solução numérica através do software ANSYS FLUENT 14.0 baseado no método dos Volumes Finitos. O algoritmo SIMPLE para acoplamento de fases foi aplicado na solução das equações discretizadas. Discretizações upwind de segunda ordem foram aplicadas para as equações de quantidade de movimento e os modelos de turbulência $\kappa$ - $\varepsilon$ foi adotado.

\section{RESULTADOS E DISCUSSÃO}

\subsection{Teste de independência da malha}

Para avaliar a independência da malha, foram selecionadas oito malhas com diferentes espaçamentos entre nós para simular o problema investigado. Distribuídas em quatro pares de mesmo tamanho de intervalo, sendo um elemento com refino na região da parede (1) e outro sem este recurso (2). Realizou-se o processo de resolução com 500 interações, o número de células empregadas, bem como o resíduo da equação da continuidade, queda de pressão obtida na simulação e desvio do valor calculado pela equação de Ergun estão apresentados na Tabela 2.

A malha B2 resultou em menores resíduo da equação da continuidade e desvio da queda de pressão em relação ao resultado obtido pela equação de Ergun (solução analítica). Levando em consideração esta análise ficou estabelecida B2 como a escolhida para a realização das investigações numéricas a serem realizadas ao longo do trabalho.

Tabela 2 - Características das malhas testadas.

\begin{tabular}{ccccc}
\hline Malha & $\begin{array}{c}\mathrm{N}^{\text {o }} \text { de } \\
\text { Células }\end{array}$ & $\begin{array}{c}\text { Resíduo } \\
\text { da } \\
\text { solução }\end{array}$ & $\begin{array}{c}\text { Queda } \\
\text { de } \\
\text { pressão } \\
(\mathrm{Pa})\end{array}$ & $\begin{array}{c}\text { Desvio } \\
\text { da Eq. } \\
\text { de } \\
\text { Ergun } \\
(\%)\end{array}$ \\
\hline A1 & 6.171 & $5,50 \mathrm{E}-02$ & 439,98 & 7,08 \\
A2 & 5.203 & $2,01 \mathrm{E}-05$ & 439,22 & 6,89 \\
B1 & 14.586 & $2,13 \mathrm{E}-05$ & 440,42 & 7,18 \\
B2 & 12.529 & $2,50 \mathrm{E}-06$ & 439,06 & 6,85 \\
$\mathrm{C} 1$ & 28.080 & $2,49 \mathrm{E}-05$ & 439,71 & 7,01 \\
$\mathrm{C} 2$ & 24.180 & $2,70 \mathrm{E}-06$ & 439,08 & 6,86 \\
D1 & 47.880 & $3,14 \mathrm{E}-05$ & 439,47 & 6,95 \\
D2 & 41.724 & $3,65 \mathrm{E}-06$ & 439,05 & 6,85 \\
\hline
\end{tabular}

Fonte: Autor (2015).

\subsection{Distribuição de Temperatura Com Porosidade de Leito Constante}

Os resultados de temperatura em função do tempo para diferentes posições axiais ao longo leito fixo são apresentada na Figura 2.

Figura 2 - Temperatura (K) no eixo central em função do tempo (min) para o meio poroso seco.

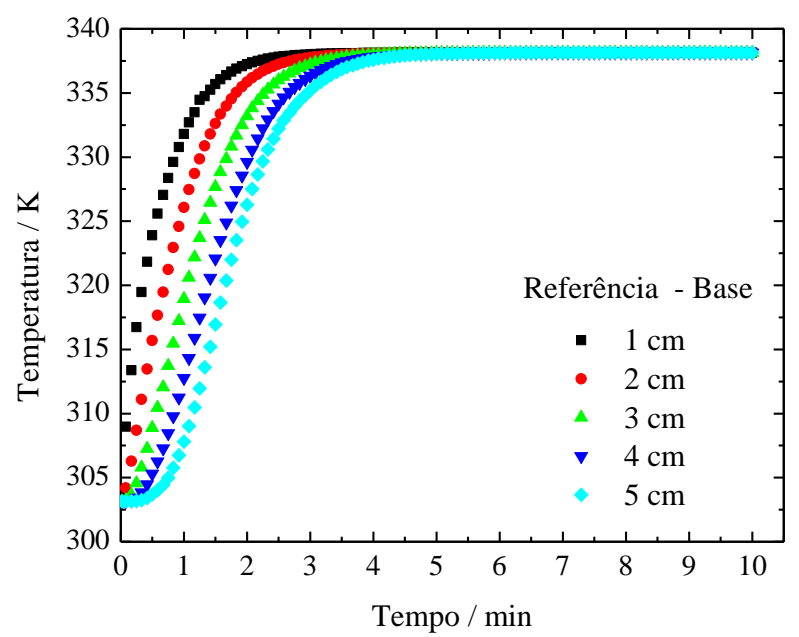

Fonte: Autor (2015).

Esses resultados foram obtidos simulando a condição de meio poroso seco $\left(\mathrm{K}_{\text {eff }}=0,27 \mathrm{~W} \mathrm{~m} \mathrm{~K}^{-1}\right)$ inicialmente $\mathrm{a}$ $303,15 \mathrm{~K}$, sendo aquecido por uma corrente de 
ar a temperatura de $338,15 \mathrm{~K}$ e velocidade de $0,98 \mathrm{~m} \mathrm{~s}^{-1}$ em condição de porosidade constante $(\varepsilon=0,40)$. Tais condições foram baseadas nos experimentos executados por Perazzini (2014), que estudou a secagem em camada fina experimentalmente, onde a velocidade e temperatura do ar foram escolhidas com o propósito de minimizar a presença da resistência externa à transferência de calor. Observa-se que a temperatura do meio poroso aumenta em regime transiente, igualando-se a temperatura do ar de aquecimento quando atinge o regime permanente. $\mathrm{O}$ tempo total para que a temperatura do meio poroso entre em equilíbrio com a temperatura de entrada do ar e a diferença verificada entre os perfis de temperatura com maior espessura são evidências do aumento da resistência ao transporte de calor quando a espessura do meio aumenta, ocorrendo de forma mais lenta na espessura de $5 \mathrm{~cm}$.

Verifica-se a concordância e comportamento semelhante das simulações ao compara-las com resultados de experimentos realizados por Perazzini (2014).

\subsection{Distribuição de Velocidade Utilizando Modelos de Porosidade}

$\mathrm{Na}$ Figura 3 são apresentados os resultados simulados da velocidade no interior do leito fixo empregando no primeiro caso porosidade constante e em cada um dos três casos seguintes um modelo de porosidade desenvolvidos por Haideger et al. (1989), Giudici (1991) e Mueller (1992).

Houve a inserção no modelo matemático por meio de UDF incorporadas ao software e simulados nas mesma condições iniciais com porosidade constante. Todos os contornos apresentados compreendem ao tempo físico de 1 minuto simulado.

Comparando-se os contornos simulados de velocidade no interior do meio poroso utilizando diferentes modelos de porosidade com os resultados obtidos inicialmente considerando a porosidade constante, verificase em todos os casos que a inserção do perfil radial de porosidade ao modelo perturbou a distribuição da velocidade no sistema.

Figura 3 - Contornos de velocidade $\left(\mathrm{m} \mathrm{s}^{-1}\right)$ para o interior do leito fixo, (A) porosidade constante, modelo de porosidade por (B) Haideger (1989), (C) Giudici (1991) e (D) Mueller (1992).
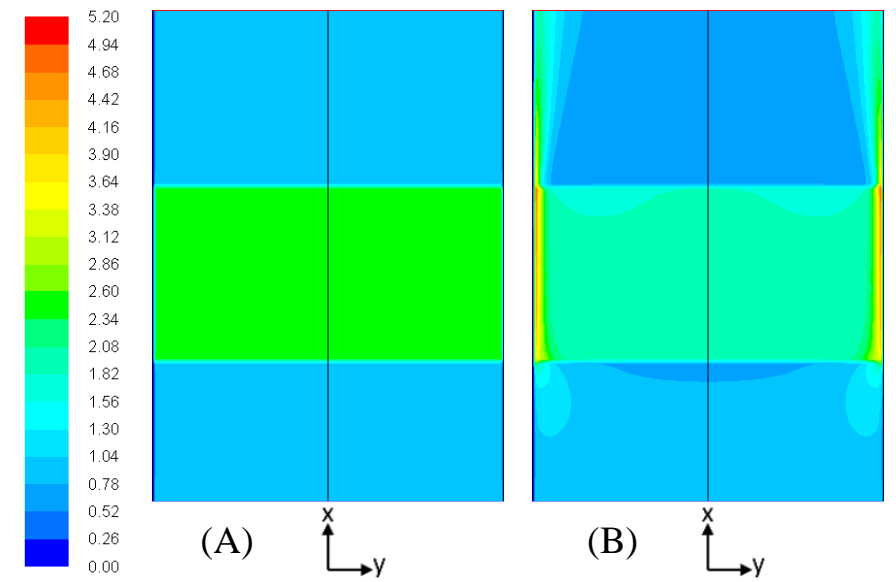

Fonte: Autor (2015).

Justifica-se esse comportamento no interior do leito e na saída em razão da maior porosidade na região próxima a parede
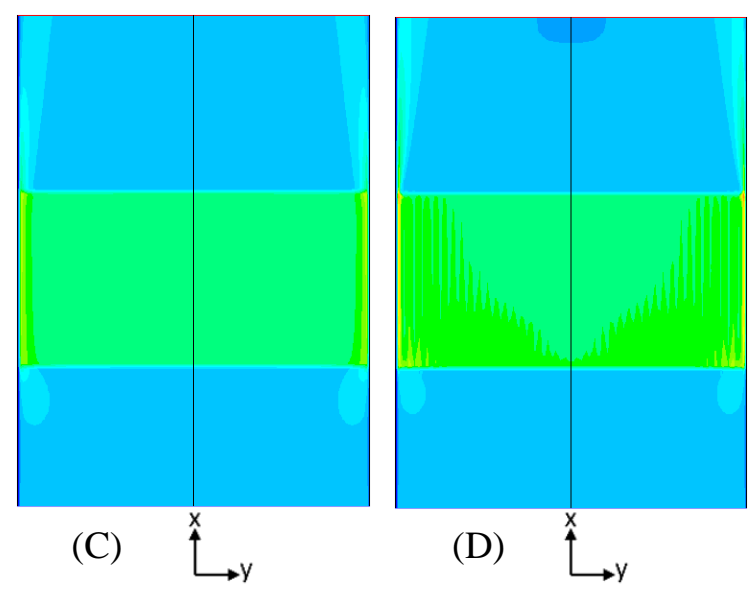

quando utilizados os modelos, devido a ocorrência de variação radial da porosidade no interior do leito fixo ocasionando um 
máximo bastante acentuado de velocidade próximo a parede (JORGE e GIUDICI, 1992).

Os resultados para o modelo de Mueller (1992) diferencia-se pela presença de um perfil oscilatório radial da velocidade, no entanto, mantendo o pico de velocidade na região da parede do leito.

Portanto, a corrente de ar perpassa preferencialmente o meio poroso na região próxima a parede devido a menor resistência a sua passagem, maior porosidade, condicionando picos de velocidade na região da parede para os três modelos testados e sem esta reação para o caso à porosidade constante. Resultados semelhantes foram observados por Béttega et al. (2013) a partir de uma abordagem análoga, entretanto, em sistema em regime permanente.

Algo que não havia sido constatado nos trabalhos recentes da literatura (NAGLIETE et al., 2010; BÉTTEGA et al., 2013) é a alteração provocada na entrada do equipamento, percebe-se nos contornos apresentados que devido ao escoamento preferencial próximo da parede há uma discreta aceleração do ar nesta região. Portanto, a variação radial da porosidade influi também no desenvolvimento do escoamento anterior ao meio poroso, esta alteração requer melhor investigação para comprovação total.

\subsection{Distribuição de Temperatura Utilizando Modelos de Porosidade}

Com intuito de avaliar a influência da variação radial da porosidade na transferência de calor a Figura 4 apresenta os contornos de temperatura no leito fixo simulado com o modelo matemático à porosidade constante $\mathrm{e}$ acrescido dos modelos de porosidade.

Figura 4 - Contornos de temperatura (K) para o interior do leito fixo, (A) porosidade constante, modelo de porosidade por (B) Haideger (1989), (C) Giudici (1991) e (D) Mueller (1992).
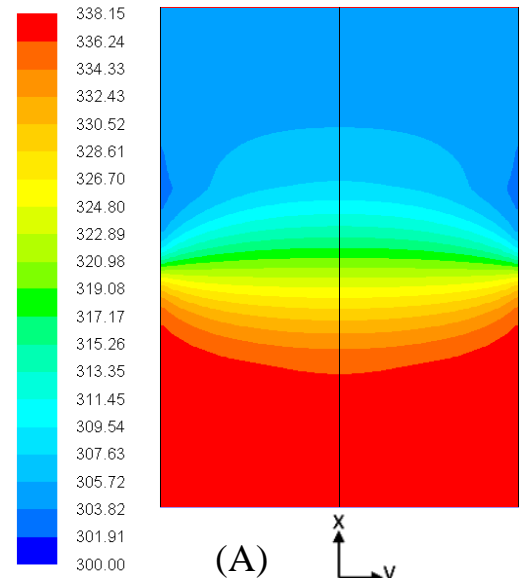

(A)

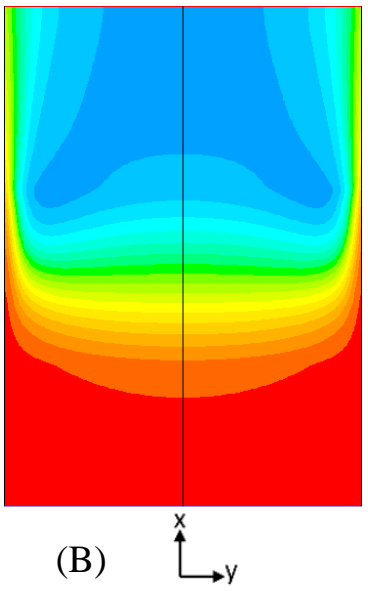

(B)

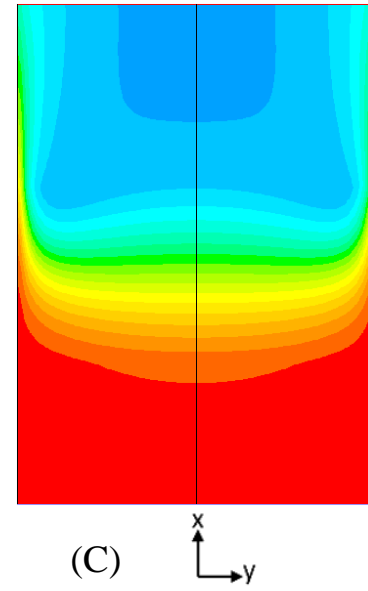

(C)

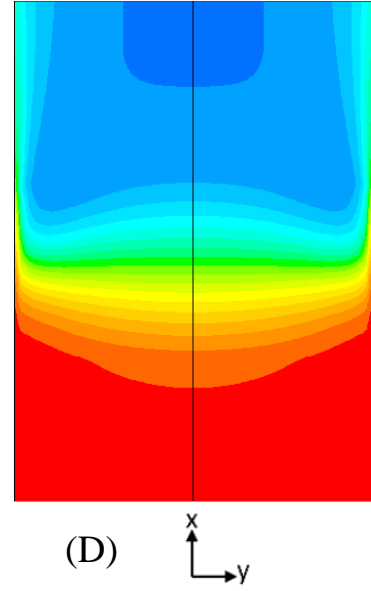

Fonte: Autor (2015).

Comparando-se os contornos simulados de temperatura no interior do meio poroso utilizando os diferentes modelos de porosidade com os resultados obtidos inicialmente considerando a porosidade constante, verifica-se que os mesmos ocasionaram perturbações em graus distintos no comportamento da temperatura tanto no interior do meio poroso como na região de saída do equipamento simulado.

A distribuição uniforme da temperatura no caso de porosidade constante foi modificada pela inserção dos modelos de porosidade, o caminho preferencial descrito nos resultados de velocidade implicaram em aquecimento mais acelerado na região 
próxima a parede, sendo gradualmente amortecido até o comportamento do caso com porosidade constante, quando a posição se aproxima do eixo central do equipamento

Béttega et al. (2012) observaram comportamentos térmicos distintos para modelos de porosidade com diferentes características. Os autores justificaram este comportamento como resultados da fluidodinâmica dos sistemas, tendo em vista que a inclusão do modelo de porosidade promove uma maior velocidade do ar na região da parede. Com maiores velocidades na região da parede, uma parcela maior da energia térmica imposta ao sistema é carregada pela corrente gasosa promovendo uma menor troca radial de calor.

Trabalhos como de Béttega et al. (2012; 2013) já indicaram que o perfil de porosidade é importante para a troca térmica no sistema, o presente estudo corrobora e amplia as informações concernentes a transferência de quantidade de movimento e calor, permitindo a obtenção de resultados pontuais de velocidade e temperatura (quantitativos) no interior do leito fixo no processo de aquecimento pela passagem de gás, portanto, caracterizando-se o regime transiente.

$\mathrm{Na}$ Figura 5 são apresentadas as temperaturas na espessura de $2,5 \mathrm{~cm}$ a partir da base em função da posição radial, os resultados obtidos através dos modelos para porosidade constante e dos modelos aplicados para a distribuição radial de porosidade utilizados neste trabalho é também possível observar que a distribuição de porosidade perturbou o comportamento da temperatura no interior do leito, conforme discutido anteriormente.

Estes resultados apresentados constituem uma fato contrário ao admitido na abordagem em camada fina, que considera o equilíbrio térmico para o gás no interior do meio poroso. Tal comportamento poderia provocar modificações também na transferência de massa durante o processo de secagem, o que necessita ser investigado, pois seria contra a premissa de equilíbrio mássico admitida na abordagem em camada fina.

Figura 5 - Temperatura na espessura de $2,5 \mathrm{~cm}$ em função da posição radial para o meio poroso seco.

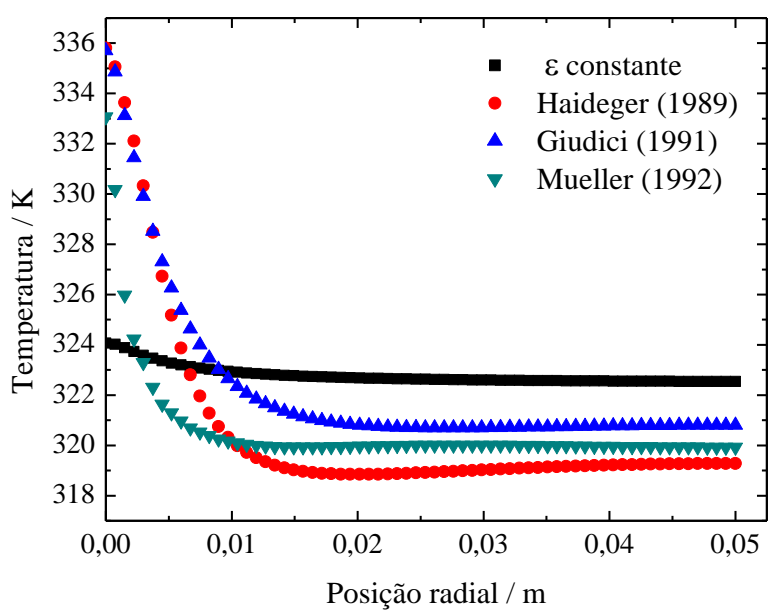

Fonte: Autor (2015).

\section{CONCLUSÃO}

A partir dos resultados obtidos no presente trabalho e posterior análise, foi possível verificar que o modelo e o procedimento numérico adotados foram apropriados para descrever a dinâmica de aquecimento e distribuição de velocidade no interior do leito fixo em todos os modelos desenvolvidos.

Constatou-se a influência da variação radial da porosidade no escoamento na região de entrada, necessitando de maior investigação para sua confirmação.

Os resultados simulados foram coerentes para as situações empregando porosidade constante e perfil de porosidade radial ao comparar-se a dados experimentais da literatura.

Os modelos de porosidade alteraram o comportamento térmico e carecem de estudo posterior para determinar sua influencia sobre a transferência de massa. 


\section{NOMENCLATURA}

$\mu$ - Viscosidade do fluido (Pa s)

$\mathrm{C}_{2}$ - Resistência inercial $\left(\mathrm{m}^{-1}\right)$

$\mathrm{Cp}$ - Calor especifico $\left(\mathrm{J} \mathrm{kg}^{-1} \mathrm{~K}^{-1}\right)$

$\mathrm{D}$ - Diâmetro do leito (m)

$\mathrm{D}_{\mathrm{p}}$ - Diâmetro da partícula $(\mathrm{m})$

$\mathrm{r}$ - Raio da partícula (m)

$\mathrm{k}_{\mathrm{eff}}$ - Condutividade térmica efetiva

$\mathrm{S}$ - Termo fonte da equação do momentum

$\mathrm{v}$ - Velocidade superficial do fluido $\left(\mathrm{m} \mathrm{s}^{-1}\right)$

$\alpha$ - Permeabilidade do meio poroso $\left(\mathrm{m}^{-2}\right)$

$\varepsilon$ - Porosidade do meio (-)

$\rho$ - Densidade do fluido $\left(\mathrm{kg} \mathrm{m}^{-3}\right)$

\section{REFERÊNCIAS}

BÉTTEGA, R.; BARROZO, M. A. S.; CORRÊA, R. G.; FREIRE, J. T. In: Congresso Brasileiro de Engenharia Química, 2012, Búzios. Anais..., 2012.

BÉTTEGA, R.; CORRÊA, R. G.; BARROZO, M. A. S.; FREIRE, J. T. XXXVI Congresso Brasileiro de Sistemas Particulados, 2013, Maceió, Anais..., 2013.

FLUENT USER'S GUIDE. Fluent Inc., Lebanon, 2009.

GIUDICI, R. Transferência de Calor em um Reator de Leito Fixo: Modelo que Incorpora Não-uniformidades Radiais no Leito. XIX Encontro sobre Escoamentos em Meios Porosos, Campinas, Anais..., pp. 262-273, 1991.

HAIDEGER, E; VORTMEYER, D. E.; WAGNER. Simultane Losuung von Energie Stoff-und Impullgleichungen fur Wandgekuhlte Chemische Fesbettreaktoren. Chem. Eng. Tech., vol 61, PP. 647-650, 1989.
KRAWCZYK, P.; BADYDA, K. Archives of Thermodynamics, vol $32, \mathrm{n}^{\circ} 4$, pp 3-16, 2011.

JORGE, L. M. M.; GIUDICI, R.; Transferência de calor em leito fixo: Modelos Alternativos. XX Encontro sobre Escoamentos em Meios Porosos, Anais..., pp. 129-140, 1992.

MUELLER, G. E.; Radial void fraction distributions in randomly packed fixed beds of uniformly sized spheres in cylindrical containers. Powder Technology, 72, pp. 269275, 1992.

NAGLIATE, F. C.; BÉTTEGA, R.; CORRÊA, R. G. In: Congresso Brasileiro de Engenharia Química, 2010. Anais..., 2010.

PERAZZINI, H.; Secagem de sólidos porosos granulares. 2014. Tese (Doutorado em Engenharia Química) - Universidade federal de São Carlos, São Carlos, 2014. 